\title{
BCCN4
}

\section{QUASI-STATIC INDENTATION AND LOW-VELOCITY IMPACT RESPONSE OF ARAMID/EPOXY COMPOSITES}

\author{
$\underline{\text { Stephanie G. Nunes }}^{(1)}$, Luís Guilherme G. Reichwald(1), Wanderley F. A. Júnior ${ }^{(2)}$, \\ Andrea Manes ${ }^{(3)}$ and Sandro C. Amico ${ }^{(1)}$
}

(1) Department of Material Engineering, Federal University of Rio Grande do Sul, Porto Alegre/RS, Brazil

(2) Department of Materials and Metallurgy, Federal University of Campina Grande, Campina Grande/PB, Brazil

(3) Department of Mechanical Engineering, Politecnico di Milano, Milan, Italy

https://doi.org/10.21452/bccm4.2018.03.01

\begin{abstract}
In applications that require impact loading, polymeric matrix composites reinforced with aramid fibers stand out, especially in relation to metals, due to characteristics such as high specific strength and stiffness, which give rise to light high-performance systems. However, such structures can fail through various modes, requiring comprehensive studies. Under lowvelocity impact, the contact time between the impactor and the target is relatively large, and the effects of deformation rate and wave propagation are generally insignificant, allowing association of the damage caused in the composites via drop-weight (DW) to those of quasistatic indentation (QSI) tests, optimizing the overall material understanding. Thus, this work aims to comparatively analyze force $\times$ displacement curves and resulting damage from dropweight and QSI tests. For that, laminates (2.5, 4.5 and $7.0 \mathrm{~mm}$ thick) were tested using variable impact energies (15, 30, 45 and $60 \mathrm{~J})$ and displacement (11, 12 and $15 \mathrm{~mm})$. Similarities in force $\times$ displacement curves and damage mechanisms between QSI and DW results were observed at the perforating threshold. However, to compare the response of the material and the damage mechanism generated by QSI and Drop-weight tests in composites, care must be taken, since there are many variables involved and the response of the composites to these tests will depend on such variables.
\end{abstract}

\section{INTRODUCTION}

Polymeric matrix composite materials, especially those reinforced with aramid fibers, are widely used since they offer attractive potential to reduce weight of high performance structures (e.g. metals) due to their high specific strength and stiffness. According to Aslan et al. [1] and Agrawal et al. [2], composite structures are more susceptible to impact damage than similar metal structures because they can fail through various failure modes and contain impact damages invisible to the naked eye, severely reducing the structural durability of the 
component. Therefore, such loads, as well as the effect they cause on these structures, are a major concern and therefore require full understanding.

Impact at low speed considerably decreases strength and stability of composite structures. Even at very low impact energy, residual damage may not be visible, especially for fiber reinforced composites of low shear stiffness. Different approaches are used to investigate the response of laminates to impact at low speed, being the drop-weight test largely used [3].

In the drop-weight impact test, a mass is raised to a known height and released, impacting the sample. In general, such event does not promote complete destruction on the target, thereby allowing residual energy to be determined by rebounding of the impactor, if necessary. The main advantage of such test over other low speed tests (e.g. Charpy and Izod) is that it allows a variety of simple and complex geometries to be tested and actual impact conditions to be simulated $[4,5]$.

In fact, a series of physical phenomena occur upon impact, such as shock and wave propagation, fracture and fragmentation, perforation and shattering [6]. In composite materials, the damage caused by low speed impact is mostly due to matrix rupture (fiber fracture is nearly non-existent) and there is a relationship between matrix rupture in the layers and delamination [7]. Low velocity impacts produce multiple delaminations in-between layers that drastically reduce their durability, strength and stiffness, even with no visible damage to the impacted surface. In fact, the energy can be absorbed at any point throughout the structure and far from the point of impact, so understanding of these damage mechanisms is very important $[8,9]$.

As the effects of the rate of deformation and wave propagation are generally negligible for low velocity impacts, quasi-static indentation test (QSI) can also be used to study the impact response of a composite material [10]. According to Nettles and Douglas [11], the QSI damage can be more easily detected, deflection of the material can be directly measured with great accuracy and the maximum transverse force can be better controlled.

In this context, this work focuses on the behavior of aramid/epoxy laminates with different thicknesses $(2.5,4.5$ and $7.0 \mathrm{~mm}$ ) subjected to quasi-static indentation and drop-weight impact tests aiming at investigating similarities in the resulting damage.

\section{MATERIALS AND METHODS}

Laminate plates of variable thicknesses $(\approx 2.5,4.5$ and $7.0 \mathrm{~mm})$ were manufactured by vacuum infusion processing, which consisted in stacking 5, 8 or 13 layers of plain-weave Kevlar $^{\circledR} 29$ fabrics (aerial weight: $440 \mathrm{~g} / \mathrm{m}^{2}$ ) on a rigid mold with a flow media partly covering the reinforcement and a vacuum bag film. After sealing the bag to the rigid mold using a sealing tape, the vacuum of 1 bar was applied into the cavity for preform compaction. Then, the epoxy system (AR $260+$ AH 260 hardener) was able to flow through the porous fabric preform, taking approximately $15 \mathrm{~min}$ to complete that. After $24 \mathrm{~h}$ of curing at room temperature, the composites were demolded and post-cured in an oven for $12 \mathrm{~h}$ at $60^{\circ} \mathrm{C}$.

The quasi-static indentation (QSI) test was performed on the laminates according to ASTM D6264, with an edge support configuration using the Instron machine, at a speed of 1.25 $\mathrm{mm} / \mathrm{min}$ (also for the return). The indenter had a hemisphere shaped tip approximately $13 \mathrm{~mm}$ in diameter and indentation was interrupted when the sample was at the perforation threshold, to obtain failure by penetration only, not perforation. The absorbed energy $\left(E_{a}\right)$ was calculated by the integral (i.e. area) of each load/unload curve using OriginPro software.

Low velocity impact tests were performed according to ASTM D7136 standard using a conventional drop-weight impact tester. Composite samples (dimensions: $100 \times 150 \mathrm{~mm}^{2}$ ) were placed between two 20-mm thick steel plates and impacted by an impactor (hardness: 60 HRC, mass: $4.4 \mathrm{~kg}$ ) with a smooth hemispherical tip approximately $16 \mathrm{~mm}$ in diameter. The impactor was instrumented with a Kistler Quartz Force Link Type 9331B $( \pm 20 \mathrm{kN})$ so that the 
impact load history could be recorded. The impact velocity, hence the energy, was measured by means of a "speed trap" comprised of two Mikroelektronik M5L/20 lasers $41 \mathrm{~mm}$ apart, which recorded the impactor crossing time. Acquisition of the signals of load cell and lasers was synchronous with a sampling frequency set to $51.2 \mathrm{kHz}$. Impact energy was varied by altering the initial velocity of the impactor (3.7-5.2 m/s), producing energy levels within 15$60 \mathrm{~J}$. Only one impact event was allowed in each sample (i.e. no rebound).

Analysis of the damages generated by the QSI and drop-weight tests in the composites was performed with the help of a Sony professional camera, model Cyber-shot GPs HD, with 20.4 MPixels of resolution and optical zoom of 50×.

\section{RESULTS}

Figure 1 shows typical force-displacement curves generated by the QSI and Drop-weight tests. The calculated values of maximum load $\left(\mathrm{F}_{\max }\right)$, maximum displacement $\left(\delta_{\max }\right)$ and absorbed energy $\left(E_{a}\right)$ are presented in Table 1. Figure 1(a) shows a second peak for the 5L30J(DW) sample due to a faulty experimental condition in which the sample was also impacted by the support of the indenter. That second peak was not used in the calculations.
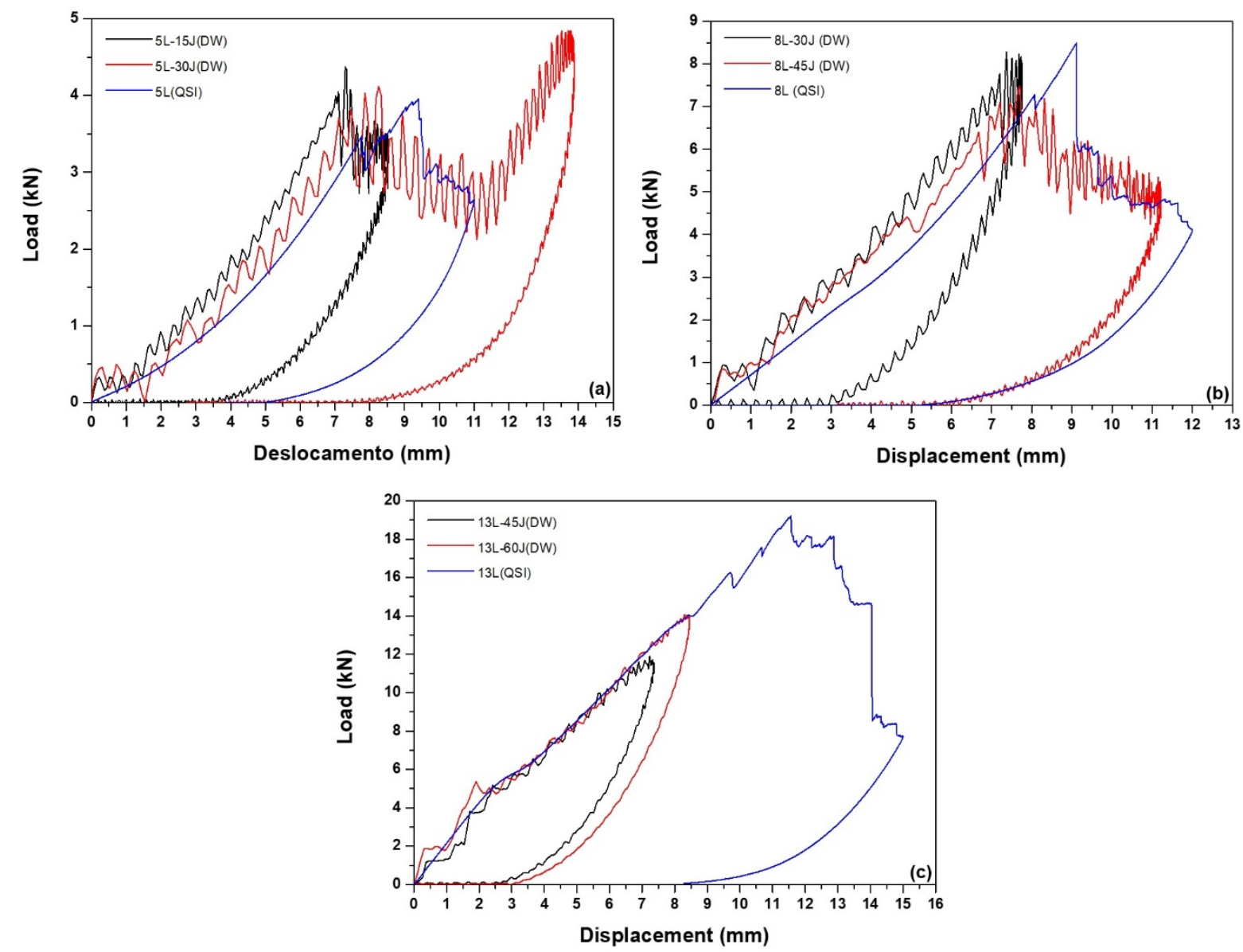

Figure 1: Force-displacement curves obtained in the drop-weight (DW) and QSI tests for the 5L (a), 8L (b) and 13L (c) composites.

In Figure 1, it is possible to observe a similarity in the behavior of the curves, with composites with 5 layers (for $15 \mathrm{~J}$ and $30 \mathrm{~J}$ ) and 8 layers (for $30 \mathrm{~J}$ and $45 \mathrm{~J}$ ) showed similar load peaks (4.4, 4.1, 8.3 and $7.5 \mathrm{kN}$, respectively), when impacted by drop-weight to those obtained by the QSI test for 5L(QSI) samples and 8L(QSI) (4.0 and $8.4 \mathrm{kN}$, respectively).

In Figure 1(a, b), it is possible to observe that the 5L-30J(DW) and $5 \mathrm{~L}(\mathrm{QSI})$ curves practically overlap, which also happens for the 8L-45J(DW) and 8L(QSI) samples. The 
greater distance between loading \& unloading phases in the curves and the high frequencies of oscillations of these curves indicate that the 5L-30J(DW) and 8L-45J(DW) samples absorbed a greater amount of energy.

In all cases, the force versus displacement graphs show closed curves, indicating that the laminates were not perforated in any of the cases and that all samples rebounded the impactor. The13L-45J(DW) sample showed he least deformation and probably the smallest damage. This is related to its stiffness, which affects the magnitude of the contact force, interfering in the extension of the damage due to the significant thickness of the composite for this impact level [2]. Also, as the 13L-60J(DW) composite was impacted with higher energy, both displacement and load (maximum peak) increased compared to the 13L-45J(DW) sample. Indeed, the limited energy available in the drop-weight test was not able to cause great damage to the $7.0 \mathrm{~mm}$ thick composite, with maximum peaks (11.9 and $14.1 \mathrm{kN}$ for 7.045J(DW) and 13L-60J(DW), respectively) lower than those for the QSI (18.9 kN). Nevertheless, the loading curve generated by QSI presents the same behavior and follows the same trend as that of the drop-weight.

Table 1: QSI and drop-weight tests results for the various laminates and energies used.

\begin{tabular}{cccc}
\hline Samples & $\mathbf{F}_{\max }(\mathbf{k N})$ & $\boldsymbol{\delta}_{\max }(\mathbf{m m})$ & $\mathbf{E}_{\mathbf{a}}(\mathbf{J})$ \\
\hline 5L-15J(DW) & 4.4 & 8.5 & 12.01 \\
5L-30J(DW) & 4.1 & 11.4 & 22.86 \\
5L(QSI) & 4.0 & 11.0 & 15.93 \\
\hline 8L-30J(DW) & 8.3 & 7.8 & 18.06 \\
8L-45J(DW) & 7.5 & 11.2 & 39.30 \\
8L(QSI) & 8.4 & 12.0 & 39.17 \\
\hline 13L-45J(DW) & 11.9 & 7.36 & 28.19 \\
13L-60J(DW) & 14.1 & 8.45 & 40.50 \\
13L(QSI) & 18.9 & 15.0 & 135.78 \\
\hline
\end{tabular}

Figures 2 and 3 present the damage occurring in the 5L (DW and QSI) and 8L (DW and QSI) composites, whose curves are more similar. The damage in the drop-weight and QSI events also showed similarity. In the rear view of the composites, an indenter cavity of similar depth is observed in Figure 2. This also happens in Figure 3 and refers to the deflection of the composites during testing, which was 11.4 and $11.0 \mathrm{~mm}$, for the 5L-30J(DW) and 5L(QSI), respectively, and 11.2 and $12.0 \mathrm{~mm}$ for composites 8L-45J(DW) and 8L(QSI), respectively.

In the cross-section view of the composites (Figure 4), samples of the same thickness showed similar mechanisms of damage, including fiber and matrix fracture, and delamination. It is also possible to observe similar sinking at the contact faces of the indenters for composites of the same thickness, and all composites are at the perforation threshold. 

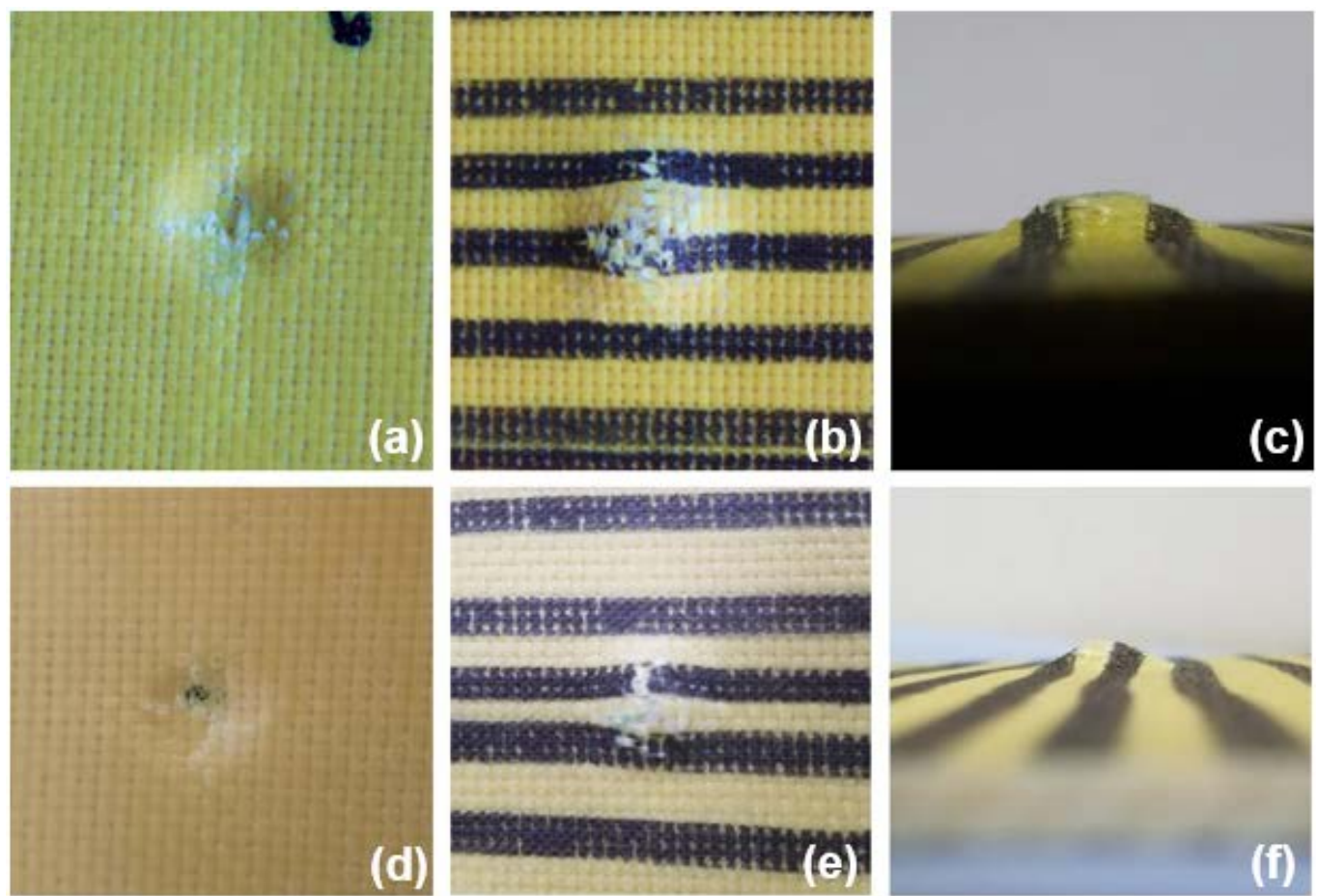

Figure 2: Front, back and side views of the damage zone for: 5L-30J(DW) (a, b, c, respectively) and 5L(QSI) (d, e, f, respectively).
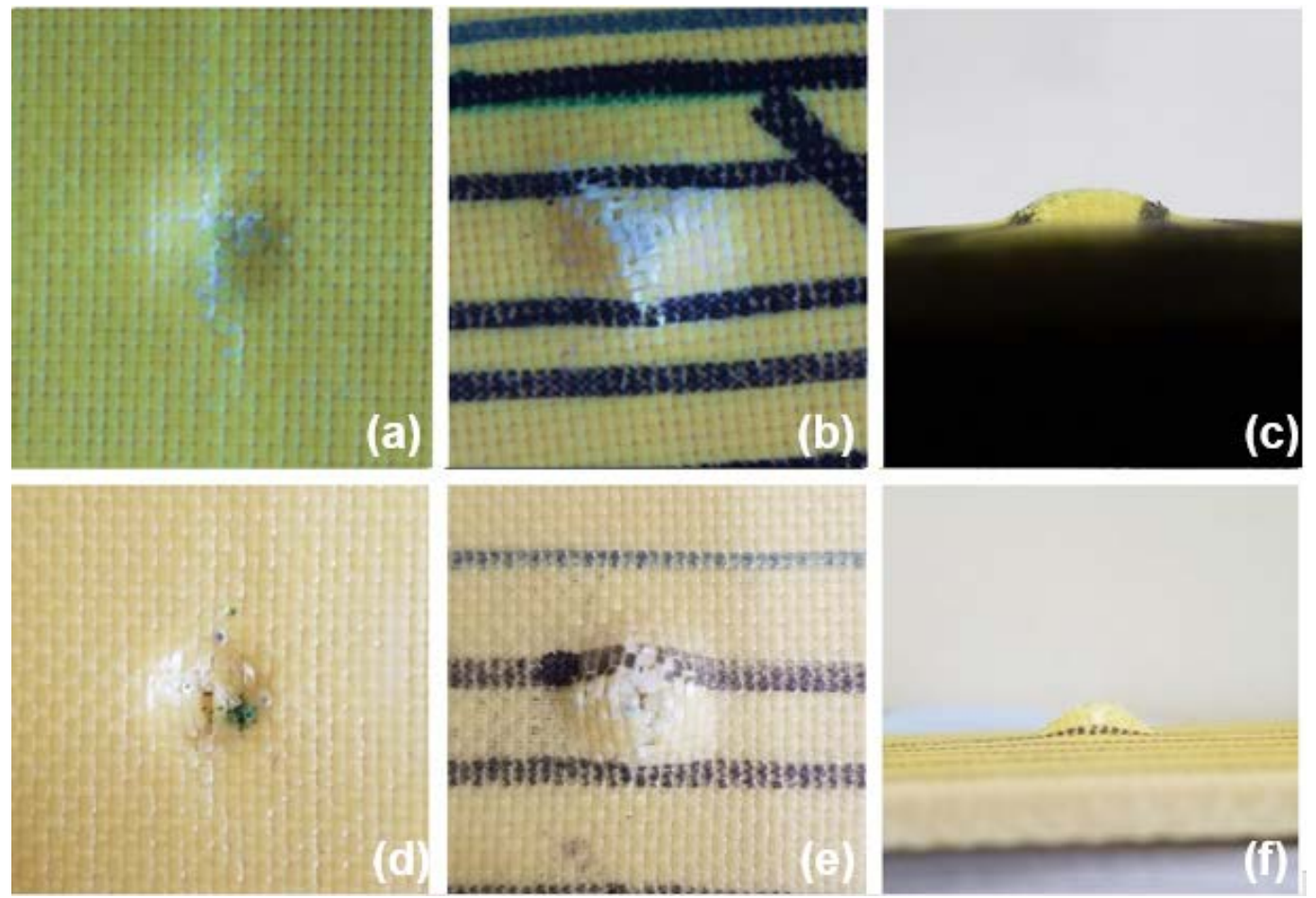

Figure 3: Front, back and side views of the damage zone for: 8L-45J(DW) (a, b, c, respectively) and 8L(QSI) (d, e, f, respectively). 

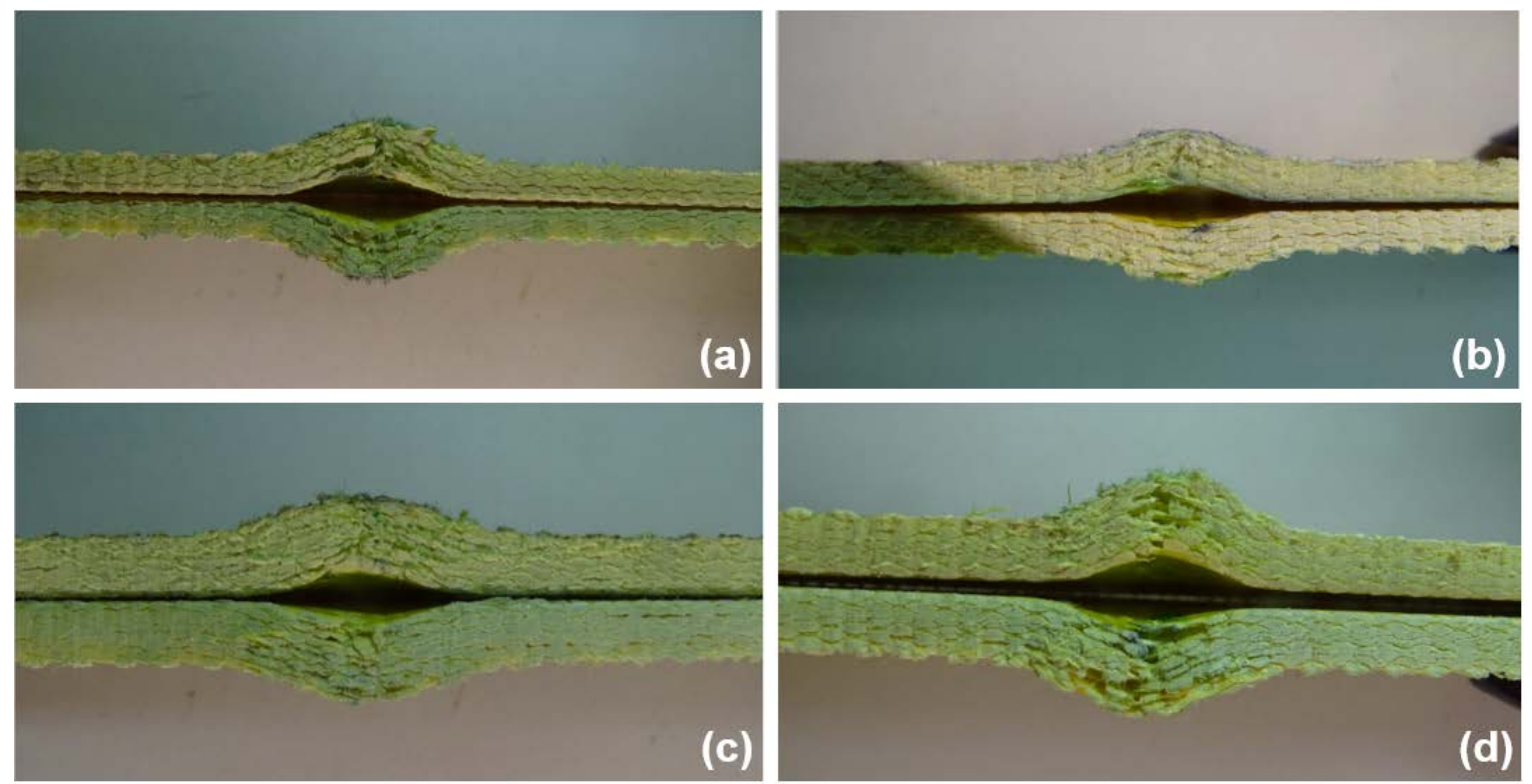

Figure 4: Cross-section view of the damage zones for: (a) 5L-30J(DW), (b) 5L(QSI), (c) 8L-45J(DW) and (d) 8L(QSI) (plates were cut in half to allow this view).

In his research comparing damage in carbon/epoxy composites submitted to QSI and dropweight tests, Guan et al. [12] reported similarity between peak loads. Regarding damage, they reported that, in general, when impact energy is low, the composite presents smaller delaminated area after drop-weight. However, at high impact energy, the delaminated area in the composite is very similar for both tests, allowing them to conclude that QSI can be used to represent a drop-weight impact event, but with some restrictions. Nettles and Douglas [11] and Lee and Zahuta [13] also state that these two tests induce similar failure mechanism, with very similar damages and force $\times$ displacement curves, for carbon/epoxy composites. On the contrary, Lawrence et al. [14] and Spronk et al. [15] reported different damages and curves from QSI and drop-weight tests for glass-S2/epoxy, carbon/epoxy and glass/polyamide composites.

\section{CONCLUSIONS}

By comparing the response of aramid/epoxy composites under QSI and drop-weight tests, it was possible to conclude that there are similarities in the force $\times$ displacement curve and damages for the samples that are at the perforating threshold (5L-30J(DW) and 5L(QSI), and 8L-45J(DW) and 8L(QSI)). This indicates that these tests can be used comparatively as long as the thicknesses is small ( 2.5 and $4.5 \mathrm{~mm}$ ), the impact energy levels are low (30 and $45 \mathrm{~J}$ ) and the samples are not perforated. However, comparison of the response and the damage mechanism generated by QSI and drop-weight tests in composites must be done with care, since there are many influencing parameters involved in these tests, such as materials (matrix and reinforcement), thicknesses, sample sizes, impactor sizes and impact energies.

\section{ACKNOWLEDGEMENTS}

The authors would like to thank DuPont ${ }^{\circledR}$ for the fiber supply and CAPES for the financial support. 


\section{REFERENCES}

[1] Aslan, Z., Karakuzu, R., Okutan, B., 'The response of laminated composite plates under lowvelocity impact loading'. Composite Structures, 59 (2003) 119-127.

[2] Agrawal, S., Singh, K. K., Sarkar, P. K., 'Impact damage on fibre-reinforced polymer matrix composite - A review', Journal of Composite Materials, 48 (3) (2014) 317-332.

[3] Duell J.M., 'Impact testing of advanced composites. Advanced Topics in Characterization of Composites’ (Michael Kessler, Canada, 2006).

[4] Cantwell, W. J.; Morton, J., 'The Impact Resistance of Composite Materials - A Review', Composites, 22 (5) (1991) 347 - 362.

[5] Safri, S. N. A.; Sultan, M. T. H.; Yidris, N.; Mustapha, F., 'Low Velocity and High Velocity Impact Test on Composite Materials - A review', The International Journal of Engineering and Science, 3 (9) (2014) 50 - 60.

[6] Razali, N.; Sultan, M. T. H., Mustapha, F.; Yidris, N.; Ishak, M. R., 'Impact Damage on Composite Structures - A Review', The International Journal of Engineering and Science, 3 (7) (2014) $08-20$.

[7] Abrate, S. 'Impact on Composite Structures' (Cambridge University Press, New York, 1998).

[8] Shyr, T; Pan, Y., 'Impact Resistance and Damage Characteristics of Composite Laminates', Composite Structures, 62 (2003) 193 - 203.

[9] Evci, C; Gulgeç, M., 'An Experimental Investigation on the Impact Response of Composite Materials', International Journal of Impact Engineering, 43 (2012) 40 - 51.

[10] Ahmed, A.; Wei, L., 'The Low-Velocity Impact Damage Resistance of the Composite Structures - A Review', Reviews on Advanced Materials Science, 40 (2015) 127 - 145.

[11] Nettles, A. T.; Douglas, M. J., 'A Comparison of Quasi-Static Indentation to Low-Velocity Impact', National Aeronautics Scape Administration (NASA), Technical Report, NASA/TP $2000-210481,2000$.

[12] Guan, Z.; Guo, Y.; Meng, Q.; Liu, D., 'Comparision of Damage Characterization of Composite Laminates Under Quasi-Static Indentation and Low-Velocity Impact', 17th International Conference on Composite Materials (ICCM17), 2009.

[13] Lee, S. M.; Zahuta, P, 'Instrumented Impact and Static Indentation of Composites', Journal of Composite Materials, 25 (1991), 204 - 222.

[14] Lawrence, B. D.; Emerson, R. P., 'A Comparison of Low-Velocity Impact and Quasi-Static Indentation’, Army Research Laboratory Technical report, ARL-TR-6272, 2012.

[15] Spronk, S. W. F.; Kersemans, M.; De Baerdemaeker, J. C. A.; Gilabert, F. A.; Sevenois, R. D. B.; Garoz, D.; Kassapoglou, C.; Van Paepegem, W., 'Comparing Damage from Low-Velocity Impact and Quasi-Static Indentation in Automotive Carbon/Epoxy and Glass/Polyamide-6 Laminates', Polymer Testing, 65 (2018) 231 - 241. 